\title{
COUPLED BUNCH MOTION IN LARGE SIZE RINGS ${ }^{\dagger}$
}

\author{
P.L. Morton, R.D. Ruth, K.A. Thompson \\ Stanford Linear Accelerator Center, Stanford, California 94309
}

\section{INTRODUCTION}

The growth of the quasi-steady-state motion of the coupled bunch oseillations in storage rings has been studied by means of a normal mode analysis to determine the beam stability. In this type of analysis, the initial amplitude displacements of the bunches are first written as a sum of the normal modes of the multiple bunch system, and then the stability of each mode is determined. If the amplitude of all modes decay tben the amplitude of all of the individual bunches must eventually decay, and the motion is considered stable. However, if the beat frequency between the different modes is sufficiently high, compared to the decay rate of the modes, it is possible for the amplitude of some of the bunches to grow temporarily before eventually decaying. Thus, even if als normal modes are eventually damped it is possible during the transient phase for the amplitude of several individual bunch oscillations to grow and become lost. Matbematical complications also arise from a moda) analysis when there is a gap in the bunch train and the wake fields from the last bunch in the train decays before arrival of the first bunch; for this case the coupled bunch motion more nearly represents that of beam breakup phenomena observed in linacs.

\section{ANALYSIS}

\section{A. Simulations Resalts}

In order to illustrate how the transient behavior can result in large amplitudes for the motion of individual bunches, even though the initial amplitudes of the individual bunches are small and the amplitude of each burich is damped, we consider the special case of a daisy chain. The daisy chain has the property that the wake field left behind by a bunch only directly affects the next bunch. This is a special case of wake fields that decay in a time short compared to the revolution period of the ring. It is convenient co number the bunches by the integer $p$ where $1 \leq p<P$ and to designate the leading bunch as number one. We denote the displacement of the pth bunch by $x_{p}$, the time derivative of the displacement by $x_{p}^{\prime}$, and assume that only the leading bunch has an initial displacement.

The model we use consists of a ring with a single cavity in which both the wake field and the damping field are produced. When a bunch passes through the cavity it receives both a wake field kick proportional to the previous bunch displacement and a damping kick, from a feedback system or radiation damping, proportional to the time derivative of the bunch motion. We use the turn number $n$ in which the bunch passes through the cavity as the time variable, i.e. $t=n T$ with $T$ the revolution period. Then passage through the cavity results in

$$
\begin{aligned}
& \Delta x_{1}^{\prime}(n)=H R x_{P}(n-1)-2 \alpha x_{1}^{\prime}(n) \\
& \Delta x_{p}^{\prime}(n)=R x_{p-1}(n)-2 \alpha x_{p}^{\prime}(n) \quad p \neq 1
\end{aligned}
$$

in which the wake field kick is given by $R$, and $H=0$ when there is a gap in the beam train and $H=1$ when the beam train is continuous. During the time between passages through the cavity the bunch oscillates about its equilibrium position $x=0$. For the case of longitudinal motion where the oscillation fre quency is much less than the revolution frequency it is not important whether the cavity is at one place in the ring or spread uniformly around the ring. Thus the difference equations of motion can be approximated by the differential equations

$$
x_{1}^{\prime \prime}(n)+2 \alpha x_{1}^{\prime}(n)+k^{2} x_{1}(n)=H R x_{p}(n-1)
$$

$$
x_{p}^{\prime \prime}(n)+2 \alpha x_{p}^{\prime}(n)+k^{2} x_{p}(n)=R x_{p-1}(n) p \neq 1
$$

in which $k=2 \pi v_{s}, v_{s}$ is the number of oscillations per revolution, and $\alpha$ is the decay rate of the oscillation amplitude.

A simulation program has been used to solve the difference equation, Eqn. 1, for the oscillation amplitudes, $a_{p}$ as a function of turn number. The amplitudes as a function of turn number are shown for the first. five bunches in Fig. 1 for three different damping rates. In Fig. 1(a) it is clear that damping rate is insufficient to prevent the transient buildup for the oscillation amplitudes for the bunches $p>4$ before they decay. However, in Figs. $1(b)$ and $1(c)$ it is not as clear whether the decay rate is sufficient to limit the transient amplitude buildup for the bunches with numbers greater than five. The values for the coupling fields and the decay rate that have been used in these simulations are much larger than would be present in an actual storage ring. If one runs the simulation for realistic values of the coupling fields and

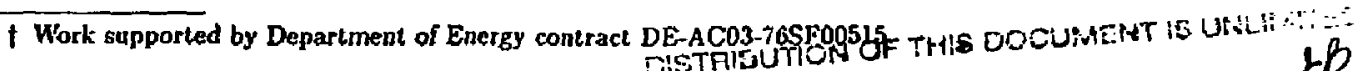


damping rates and for a sufficient number of bunches the maximum amplitude of each bunch can be determined, but the number of turns that we must run to find the maximum amplitudes of all $P$ bunches goes like $P / \alpha$ which places a burden on the compuler. We present below an analytic method that one can use to find the maximum amplitude and the turn number when it occurs. The circles shown on Fig, 1 are the maximum amplitude of each bunch and the turn number when it occurred as given by the analytic calculation below.

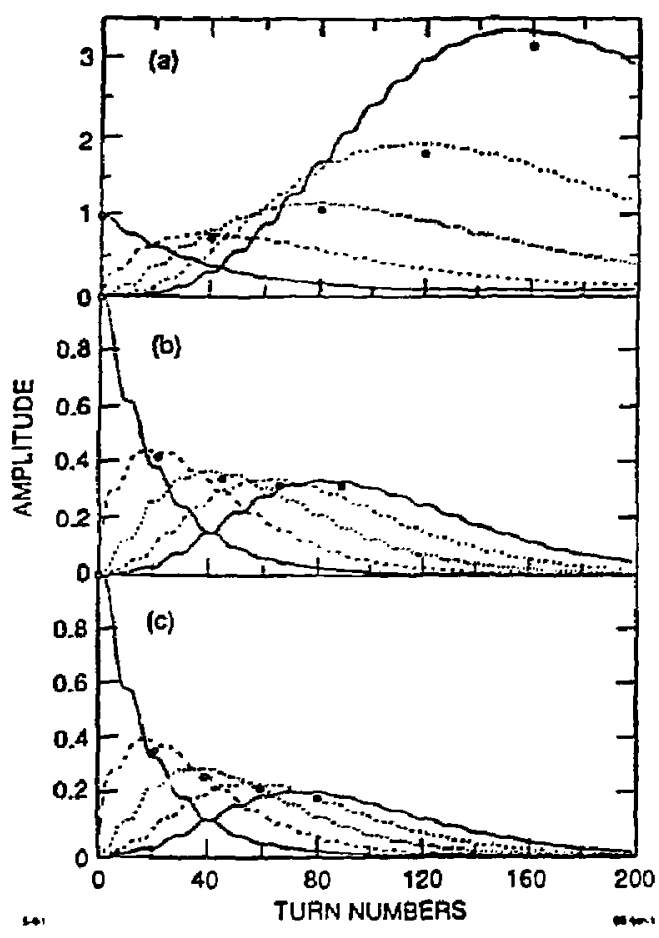

Figure 1. Oscillation amplitudes of the first five bunches versus turn number for $R / k=0.1$ and $\nu=0.05$. l(a) $\alpha=0.025,1(b) \alpha=0.045$, and $I(c) \alpha=0.05$ Circles represent analytic calculation of the maximum amplitudes.

\section{B. Analytic Results}

We write for the bunch displacement

$$
x_{p}(n)=y_{p}(n) e^{-a n} e^{i k n}
$$

with $\bar{k}^{2}=k^{2}-\alpha^{2}$. We use the slowly varying amplitude and phase approximation in which we ignore $y_{p}^{\prime \prime}$ compared to $k y_{\mathrm{g}}^{\prime}$ to obtain

$$
y_{p+1}^{\prime}=\frac{-i R}{2 t} y_{p}
$$

where we also have assumed that $\alpha^{2}<k^{2}$ so that $\bar{k} \approx k$.

At turn $n=0$ we have the following initial conditions: $y_{1}=c_{2}, y_{2}^{\prime}=0$ and $y_{p}=y_{p}^{\prime}=0$ for $p \neq 1$. The solution to Eqns. 3 and 4 is given by

$$
x_{p+1}=\frac{a_{1}}{p !}\left(\frac{-i R n}{2 k}\right)^{p} e^{-a_{m}} e^{i k n} .
$$

The amplitude of the oscillation $a_{p}$ may be written as

$$
a_{p}=\sqrt{x^{2}+\left(x_{p}^{\prime} / t\right)^{2}}=\sqrt{x_{p} x_{p}^{*}}
$$

or

$$
a_{p+1}=\frac{a_{1}}{p !}\left(\frac{R n}{2 k}\right)^{p} e^{-a n} .
$$

From Eqn. 7 we find that the maximum amplitude $\widehat{a}_{p+1}$ and the turn when the maximum occurs $\bar{n}$ are given by

$$
\hat{a}_{p+i}=\frac{a_{1}}{p !}\left(\frac{R p}{2 L a}\right)^{p} e^{-p}
$$

and

$$
\tilde{n}=\left(\frac{p}{a}\right) .
$$

The values of the coupling and the decay rate used is: the simulations for Fig. 1 have been substituted into Eqn. $\delta$ and the results are plocted in Figs. $I$ and 2.

Finding the maximum oscillation amplitudes from a simulation program requires one to run the program for the number of turns in a damping period tirnes 21. number of bunches in the ring which, for large malia ple bunch colliders, is of the order of millions of curns

We note that the inaximum amplitude of $\bar{a}_{p}$ in always bounded, but for large values of $\mu$ the max. inum amplitude can become quile large after many turns. Clearly, in a practical ring sorne restiction on the maximum amplitude for all bunches must bo so. quired. We specify that the coupled bunch motion will be acceptable if all oscillation amplitiddes of th. bunches are less than the intial amplitude of the first bunch, i.e. $\hat{a}_{p} \leq a_{1}$. This requires that 


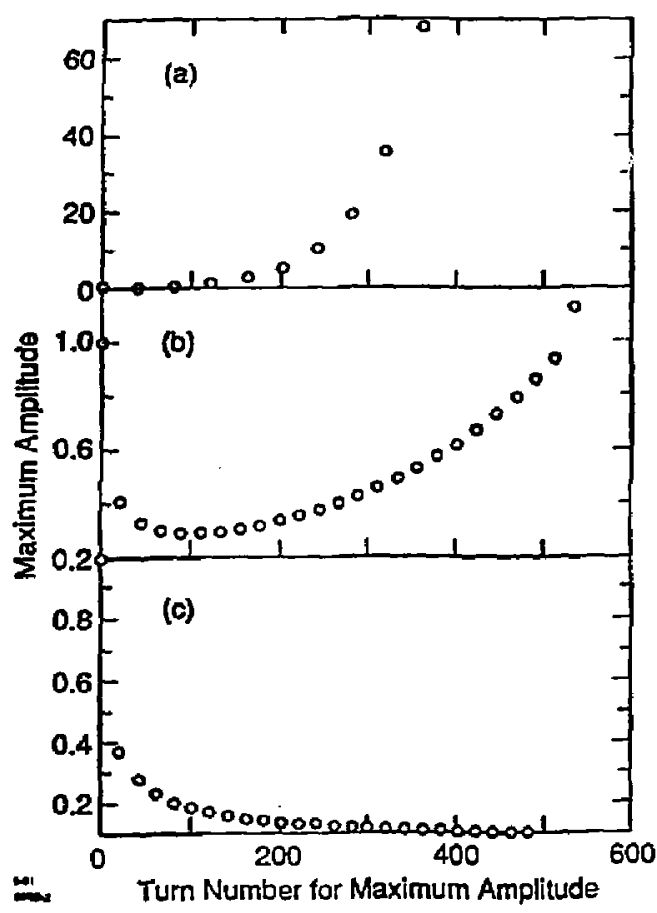

Figure 2. Maximum amplitudes of the bunches versus turn number when maximum occurs. 2(a) $\alpha=0.025$, 2(b) $\alpha=0.045$, and 2 (c) $a=0.05$.

$$
\left(\frac{R p}{2 k \alpha}\right) e^{-1} \leq\left(\frac{1}{p !}\right)^{\frac{1}{p}}
$$

If we use Stirlings formula for large $p$ we have $\left(\frac{1}{p !}\right)^{\frac{1}{p}} \approx$ $p e^{-1}$ which yields the following criteria for the required damping rate

$$
\alpha \geq \frac{R}{2 k}
$$

for acceptable motion in the sense that $\hat{a}_{p} \leq a_{1}$ for all $p$.

\section{Normal Mode Analysis}

For the case where the bunch train is continuous $(H=1)$ it is easy to solve Eqn. 2 for $\varepsilon_{m}(n)$, the amplitude of the mth normal mode. We find that the displacement of the pth bunch for the mth mode can be approximated by

$$
x_{p}^{(m)}(n)=\xi_{m}(0) e^{\frac{(\{2 \pi m+k) \psi}{p}} e^{\left(\gamma_{m}-\alpha\right) n}
$$

where again we have assumed that $k \gg \gamma_{m}$ and $k>\alpha$, so that $\gamma_{m}$ is given by

$$
\Re e\left(\gamma_{m}\right)=\frac{R}{2 k} \sin \left(\frac{2 \pi m+k}{P}\right)
$$

and

$$
\operatorname{Sm}\left(\gamma_{m}\right)=-\frac{R}{2 k} \cos \left(\frac{2 \pi m+k}{P}\right) .
$$

The modes which are stable have $\Re e\left(\gamma_{m}-\alpha\right) \leq 0$, which yields the condition for stability. of all of the modes

$$
\alpha \geq \frac{R}{2 k}
$$

This condition is exactly the same condition as we obtained in the transient case (Eqn. 10) when only the first bunch in the train had an initial displacement. 